ARTICLE

https://doi.org/10.1038/s41467-019-08363-2

\title{
Infrared-pump electronic-probe of methylammonium lead iodide reveals electronically decoupled organic and inorganic sublattices
}

\author{
Peijun Guo (1) 1, Arun Mannodi-Kanakkithodi', Jue Gong', Yi Xia', Constantinos C. Stoumpos (1) ${ }^{3}$, Duyen H. Cao ${ }^{4}$, \\ Benjamin T. Diroll', John B. Ketterson" ${ }^{5}$, Gary P. Wiederrecht ${ }^{1}$, Tao Xu (1) ${ }^{2}$, Maria K.Y. Chan?', \\ Mercouri G. Kanatzidis ${ }^{3}$ \& Richard D. Schaller (1) 1,3
}

Organic-inorganic hybrid perovskites such as methylammonium lead iodide $\left(\mathrm{CH}_{3} \mathrm{NH}_{3} \mathrm{Pbl}_{3}\right)$ are game-changing semiconductors for solar cells and light-emitting devices owing to their defect tolerance and exceptionally long carrier lifetimes and diffusion lengths. Determining whether the dynamically disordered organic cations with large dipole moment benefit the optoelectronic properties of $\mathrm{CH}_{3} \mathrm{NH}_{3} \mathrm{Pbl}_{3}$ has been an outstanding challenge. Herein, via transient absorption measurements employing an infrared pump pulse tuned to a methylammonium vibration, we observe slow, nanosecond-long thermal dissipation from the selectively excited organic mode to the inorganic sublattice. The resulting transient electronic signatures, during the period of thermal-nonequilibrium when the induced thermal motions are mostly concentrated on the organic sublattice, reveal that the induced atomic motions of the organic cations do not alter the absorption or the photoluminescence response of $\mathrm{CH}_{3} \mathrm{NH}_{3} \mathrm{Pbl}_{3}$, beyond thermal effects. Our results suggest that the attractive optoelectronic properties of $\mathrm{CH}_{3} \mathrm{NH}_{3} \mathrm{Pbl}_{3}$ mainly derive from the inorganic lead-halide framework.

\footnotetext{
${ }^{1}$ Center for Nanoscale Materials, Argonne National Laboratory, 9700 South Cass Avenue, Lemont, IL 60439, USA. ${ }^{2}$ Department of Chemistry and Biochemistry, Northern Illinois University, 1425 W. Lincoln Hwy., DeKalb, IL 60115, USA. ${ }^{3}$ Department of Chemistry, Northwestern University, 2145 Sheridan Road, Evanston, IL 60208, USA. ${ }^{4}$ Materials Science Division, Argonne National Laboratory, 9700 South Cass Avenue, Lemont, IL 60439, USA. ${ }^{5}$ Department of Physics and Astronomy, Northwestern University, 2145 Sheridan Road, Evanston, IL 60208, USA. Correspondence and requests for materials should be addressed to R.D.S. (email: schaller@anl.gov)
} 
$\mathrm{M}$ etal-halide perovskites are emerging as low-cost, solution-processable semiconductors for applications involving the harvesting and emitting of light ${ }^{1,2}$. Recent spectroscopic measurements have demonstrated that the favorable optical and electronic properties of these materials likely arise from the strong electron-phonon interactions in these soft semiconductors, in conjunction with the large dynamic disorder of the lead-halide octahedral framework ${ }^{3-6}$. Hybrid organic-inorganic metal-halide perovskites, including the prototypical $\mathrm{CH}_{3} \mathrm{NH}_{3} \mathrm{PbI}_{3}$, present additional challenges in understanding the interplay between the structural and optoelectronic properties. This primarily stems from the significant structural fluctuations of the organic cations (e.g., $\mathrm{CH}_{3} \mathrm{NH}_{3}{ }^{+}$) with picosecond relaxation times observed from neutron scattering and millmeter-wave spectroscopic experiments ${ }^{7,8}$. Due to the potentially crucial importance for enhancing device efficiency ${ }^{9}$ and enabling additional functionalities ${ }^{10,11}$, the mutual interactions between the fluctuating dipolar organic cations and the charge carriers that reside on the inorganic $\mathrm{Pb}-\mathrm{I}$ framework have been extensively investigated. Formation of (anti)ferroelectric domains arising from the orientational ordering of the polar organic cations was proposed to enhance carrier lifetimes ${ }^{12}$, but spectroscopic measurements did not support such a picture ${ }^{13-15}$. Dynamic bulk Rashba effect induced by the $\mathrm{CH}_{3} \mathrm{NH}_{3}{ }^{+}$cations was also suggested ${ }^{16,17}$, yet recent theoretical and experimental results show that such an effect is negligible ${ }^{18}$. In fact, HC $\left(\mathrm{NH}_{2}\right)_{2} \mathrm{PbI}_{3}$-based devices exhibit conversion efficiencies comparable to the $\mathrm{CH}_{3} \mathrm{NH}_{3} \mathrm{PbI}_{3}$ analog ${ }^{19}$, albeit the dipole moment of $\mathrm{HC}\left(\mathrm{NH}_{2}\right)_{2}{ }^{+}$is much smaller than that of $\mathrm{CH}_{3} \mathrm{NH}_{3}{ }^{+}$(ref. ${ }^{12}$ ), and approaches that of the non-polar $\mathrm{Cs}^{+}$cation. Although spectroscopy-based comparative studies between hybrid and allinorganic perovskites have been attempted so as to evaluate the role of polar organic cations on material performance ${ }^{20-22}$, the properties of metal-halide perovskites are known to depend on synthetic conditions and material history ${ }^{23,24}$. For example, highquality, vapor-deposited $\mathrm{CsPbI}_{3}$ thin films present impressively long carrier lifetime exceeding $10 \mu$ s that is comparable to the best $\mathrm{CH}_{3} \mathrm{NH}_{3} \mathrm{PbI}_{3}$ counterparts, whereas solution-processed $\mathrm{CsPbI}$ film showed significantly shorter lifetime ${ }^{25}$. Remarkable solar cell efficiencies derived from carefully prepared $\mathrm{CsPbI}_{3}$ have been reported ${ }^{26,27}$, and solar cells made from $\mathrm{CH}_{3} \mathrm{NH}_{3} \mathrm{PbBr}_{3}$ and $\mathrm{CsPbBr}_{3}$ exhibited comparable efficiencies ${ }^{21}$. These device characteristics hinted that the main role played by the organic cations is structural, with a different size than $\mathrm{Cs}^{+}$as well as the additional electrostatic interactions and hydrogen bonding with the lead-halide framework ${ }^{28}$. While the black phase of $\mathrm{CH}_{3} \mathrm{NH}_{3} \mathrm{PbI}_{3}$ is stable at room temperature, it is known that the $\mathrm{CsPbI}_{3}$ counterpart is not stable at room temperature and special treatments are required to stabilize its black phase ${ }^{29}$, which makes a fair comparison between $\mathrm{CH}_{3} \mathrm{NH}_{3} \mathrm{PbI}_{3}$ and $\mathrm{CsPbI}_{3}$ difficult. As a result, experimental approaches permitting the direct probing of interactions between the organic and inorganic sublattices are important for the study of hybrid material systems ${ }^{30}$. Here, we employ infrared pump electronic-probe (IPEP) spectroscopy to excite the strongly absorbing vibrational modes of the organic sublattice and examine the corresponding optical response near the bandgap of $\mathrm{CH}_{3} \mathrm{NH}_{3} \mathrm{PbI}_{3}$. We found that the pump-induced motions of the $\mathrm{CH}_{3} \mathrm{NH}_{3}{ }^{+}$cations do not change the subsequent absorption or the photoluminescence response of $\mathrm{CH}_{3} \mathrm{NH}_{3} \mathrm{PbI}_{3}$, but merely result in the heating of the inorganic sublattice in hundreds of picoseconds to nanosecond timescale.

\section{Results}

Static optical response. Figure 1a maps the temperaturedependent static absorbance of a $\mathrm{CH}_{3} \mathrm{NH}_{3} \mathrm{PbI}_{3}$ thin film around its bandgap. The tetragonal-to-orthorhombic phase transition near $145 \mathrm{~K}$ is denoted by an abrupt change of the bandgap, and
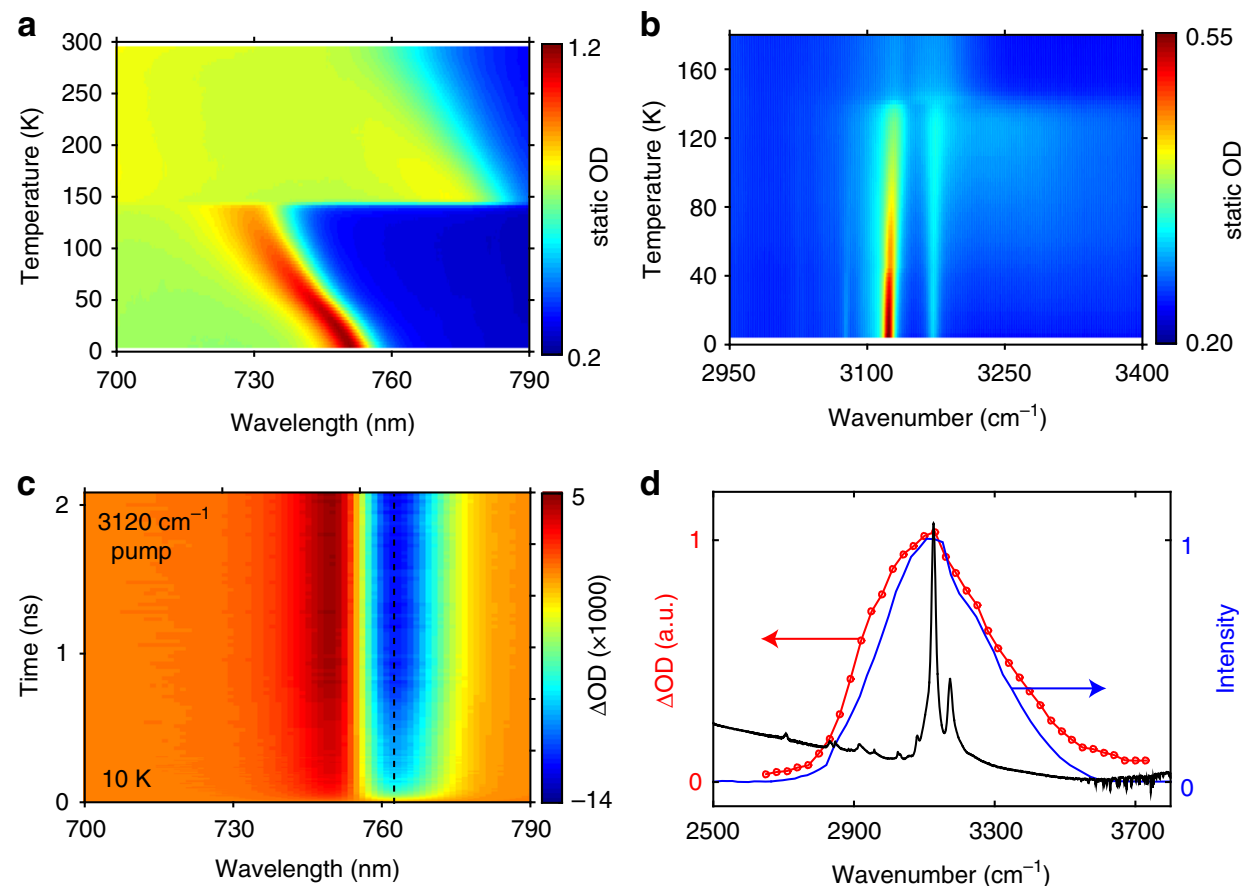

d

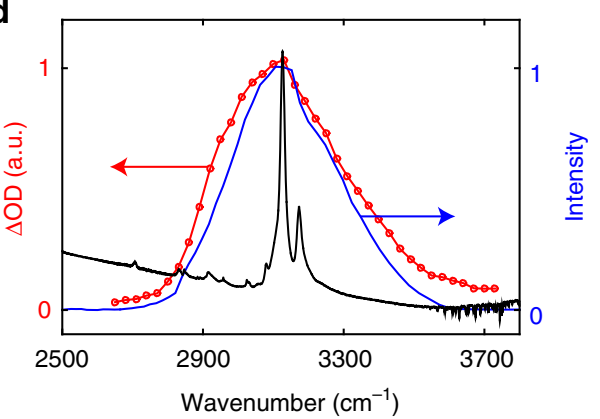

Fig. 1 Static and infrared pump electronic-probe (IPEP) measurements of $\mathrm{CH}_{3} \mathrm{NH}_{3} \mathrm{Pbl}_{3}$. a Static, temperature-dependent absorbance around the bandgap. b Static, temperature-dependent absorbance around the $\mathrm{N}-\mathrm{H}$-as vibrational modes. c $\Delta \mathrm{OD}$ spectral map measured with on-resonance infrared pump (centered at $3120 \mathrm{~cm}^{-1}$ ) at $10 \mathrm{~K}$. The dashed line shows the wavelength at which the kinetics are extracted (Fig. 2b). d Red: dependence of the magnitude of the strongest bleach signal on the center wavenumber of the pump pulse with a fixed fluence (measured at $10 \mathrm{~K}$ ). Blue: spectral profile of the pump pulse centered at $3120 \mathrm{~cm}^{-1}$. Black: static absorbance of $\mathrm{CH}_{3} \mathrm{NH}_{3} \mathrm{Pbl}_{3}$ (in arbitrary units) at $80 \mathrm{~K}$ 

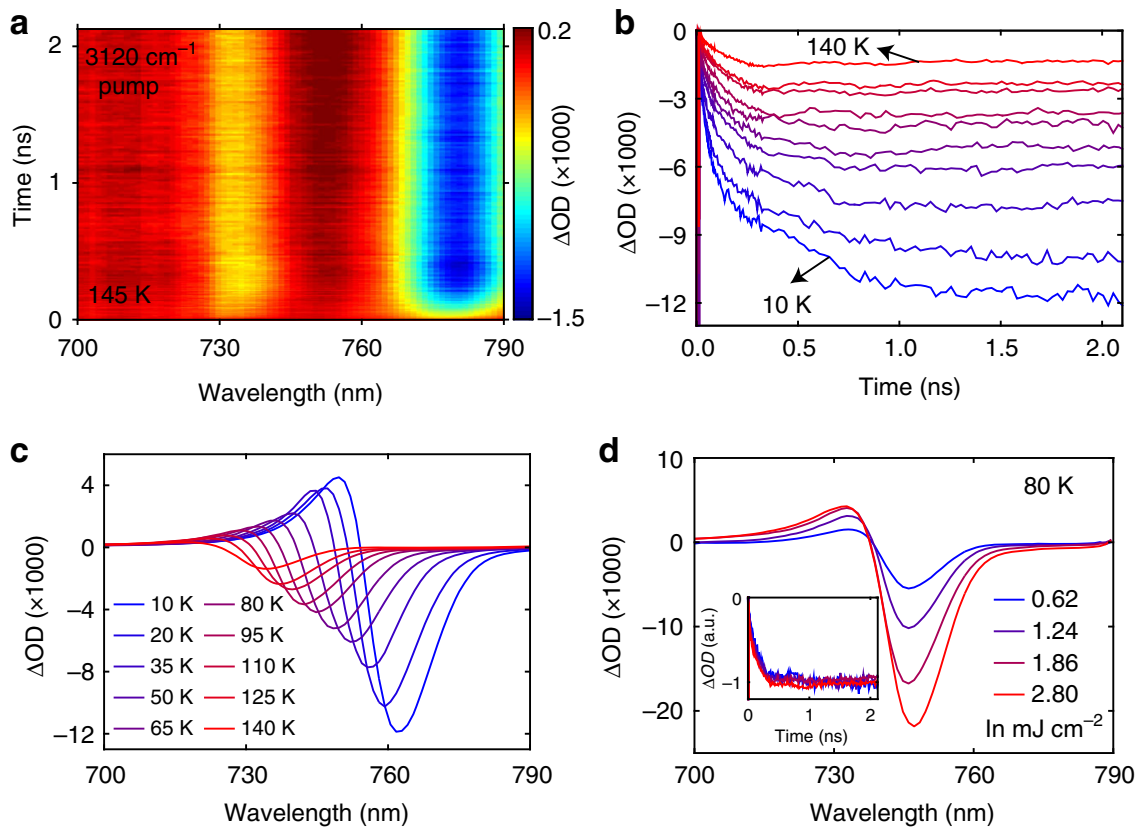

Fig. 2 Temperature- and fluence-dependent transient optical response. a $\Delta O D$ spectral map measured at $145 \mathrm{~K}$ with on-resonance infrared pump. $\mathbf{b}$ Transient kinetics at the wavelength that shows the strongest bleach signal (indicated by the dashed line in Fig. 1c), measured at different temperatures. c Transient spectra at $2 \mathrm{~ns}$ delay time measured at different temperatures. Legend in (c) applies to (b) as well. Pump fluence used in (b, c) was $0.45 \mathrm{~mJ} \mathrm{~cm}^{-2}$. d Fluence-dependent transient optical spectra at 2 ns delay time; inset: kinetics for different fluences appear nearly unchanged

for each individual phase the bandgap increases with temperature. The near-bandgap, distinct absorption peak in the orthorhombic phase arises due to a comparatively stronger excitonic character. Figure $1 \mathrm{~b}$ presents the temperature-dependent static absorbance arising from the $\mathrm{N}-\mathrm{H}$ asymmetric stretching modes (abbreviated here as $\mathrm{N}-\mathrm{H}$-as modes) of the $\mathrm{CH}_{3} \mathrm{NH}_{3}{ }^{+}$cation, which exhibit the strongest optical absorption cross-section among various vibrational modes of the molecular cation ${ }^{31}$. A dramatic increase of the $\mathrm{N}-\mathrm{H}$-as absorbance accompanying the phase transition is due to the formation of hydrogen bonding that suppresses the rotational motions of $\mathrm{CH}_{3} \mathrm{NH}_{3}{ }^{+}$in the orthorhombic phase ${ }^{32}$. The pronounced mid-infrared absorbance, in conjunction with the near-bandgap optical response, facilitate IPEP measurements on the orthorhombic phase of $\mathrm{CH}_{3} \mathrm{NH}_{3} \mathrm{PbI}_{3}$.

IPEP measurements. In IPEP measurements, we selectively pumped the N-H-as modes (centered at $3120 \mathrm{~cm}^{-1}$; Fig. $1 \mathrm{~d}$ and Supplementary Figure 1 show the pump profile) and probed the change of optical density $(\Delta \mathrm{OD})$ near the bandgap. The $\Delta \mathrm{OD}$ spectral map acquired on $\mathrm{CH}_{3} \mathrm{NH}_{3} \mathrm{PbI}_{3}$ at $10 \mathrm{~K}$ is displayed in Fig. 1c. We observe a derivative-like transient spectral response, whose magnitude following the excitation starts from zero, grows with pump-probe time delay, and saturates on the nanosecond timescale. Positive $\triangle O D$ signal on the blue side of the static absorption peak and negative signal to the red conveys a spectral blueshift, consistent with transient lattice heating (Fig. 1a). Note that large bandgap shifts arising from the optical Stark effect were observed at zero delay time, when the pump and probe pulses temporally overlapped ${ }^{33}$ (Supplementary Fig. 2). As presented in Fig. 1d, sweeping the frequency of the infrared pump pulse shows that the obtained intensity of $\Delta \mathrm{OD}$ is linearly proportional to the spectral overlap of the pump and the absorbance of the $\mathrm{N}$-H-as modes. Furthermore, an off-resonance pump (centered at $2200 \mathrm{~cm}^{-1}$ ) results in negligible transient response (Supplementary Fig. 3), confirming that the response shown in Fig. 1c is due to vibrational excitation of the organic sublattice. The nanosecond timescale relates slow down-conversion of thermal energy from the high-energy $\mathrm{N}-\mathrm{H}$-as modes to the low-lying, cold phonon bath, with the latter encompassing all the $\mathrm{CH}_{3} \mathrm{NH}_{3}{ }^{+}$ modes except the N-H-as modes, and all the modes of the inorganic sublattice.

Probing the tetragonal phase at $205 \mathrm{~K}$ distills a comparably faster thermal equilibration with a timescale of hundreds of picoseconds (Supplementary Fig. 4). Measurement near the phase transition (near $145 \mathrm{~K}$ ) reveals that the two coexisting phases respond independently to the pump (Fig. 2a), as no evidence of transient phase change is observed, which would otherwise lead to the transfer of spectral weight between the two phases. The $\triangle \mathrm{OD}$ amplitude near the bandgap obtained for the tetragonal phase is, as expected, weaker than that for the orthorhombic counterpart, owing to a weaker vibrational absorption (Fig. 1b). The nearly identical timescales of $\Delta \mathrm{OD}$ kinetics for the coexisting phases at $145 \mathrm{~K}$ (Supplementary Fig. 4d) indicate that the rotations of $\mathrm{CH}_{3} \mathrm{NH}_{3}{ }^{+}$, which are only present in the tetragonal phase, do not apparently influence the phonon-phonon coupling strength between the two sublattices. Temperature-dependent kinetics (Fig. 2b) reveal slower thermal equilibration at lower temperatures. Furthermore, the $\triangle \mathrm{OD}$ amplitude increases with decreasing temperature (Fig. 2c), which results from the heat capacity that diminishes, and the absorption cross-section of the $\mathrm{N}-\mathrm{H}$-as modes that grows, with decreasing temperature. Fluencedependent measurements (Fig. $2 \mathrm{~d}$ ) show that the $\triangle \mathrm{OD}$ amplitude scales nearly linearly with the pump fluence, and the thermal equilibration time is not sensitive to pump fluence over the explored range, consistent with earlier work based on transient mid-infrared spectroscopy using electronic excitation and an infrared probe ${ }^{31}$.

Having established the transient electronic response around the bandgap following infrared molecular vibrational excitation, we then turn to investigating whether perturbatively induced motions of the organic sublattice influence the near-bandgap optical properties of $\mathrm{CH}_{3} \mathrm{NH}_{3} \mathrm{PbI}_{3}$. As illustrated in Fig. 3a, 

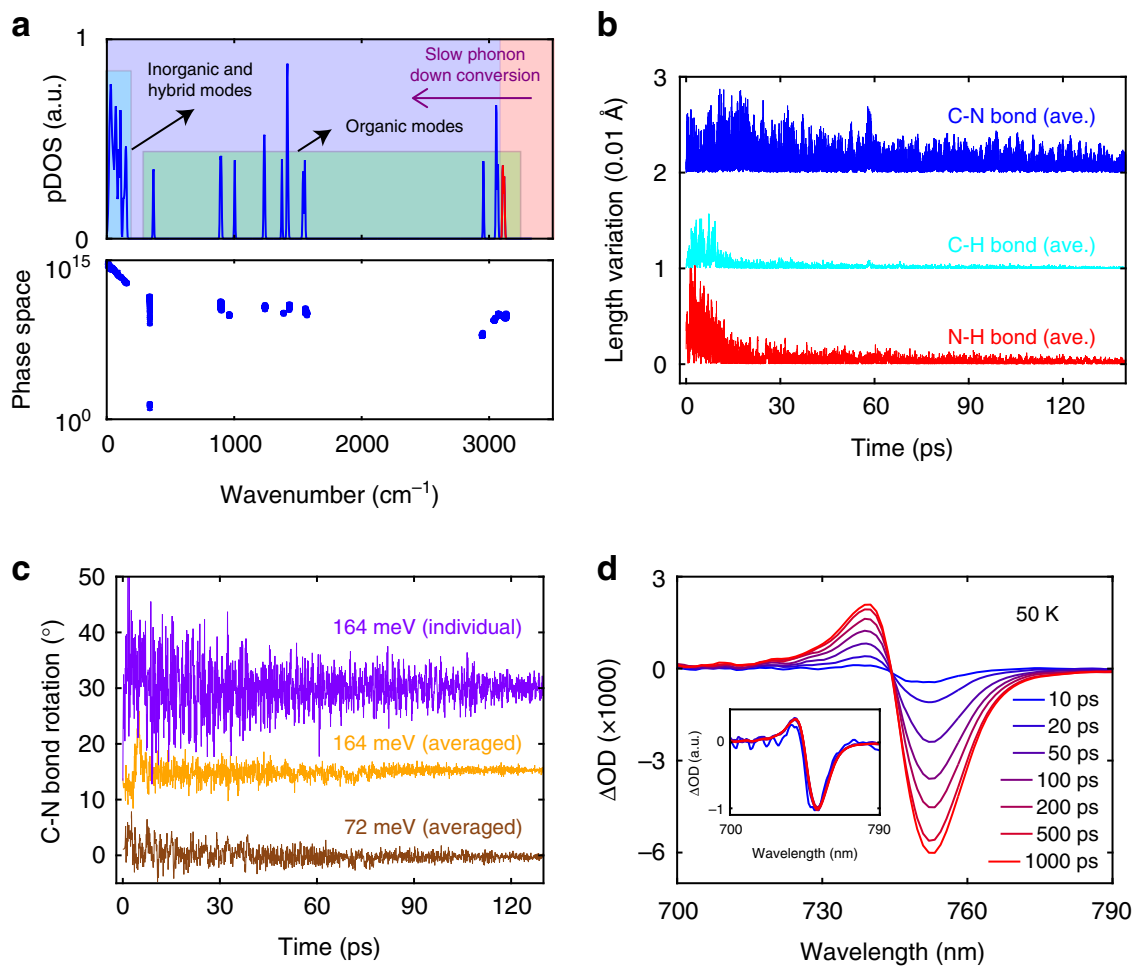

Fig. 3 First-principles calculation of the phonon dynamics for orthorhombic $\mathrm{CH}_{3} \mathrm{NH}_{3} \mathrm{Pbl}$. a Calculated zero-Kelvin phonon density of states (pDOS) and phonon scattering phase space. $\mathbf{b}$ Absolute variation in the $\mathrm{N}-\mathrm{H}$ (red), $\mathrm{C}-\mathrm{H}$ (cyan), and C-N (blue) bond lengths from ab initio molecular dynamics (AIMD) simulations. Data are averaged over the four $\mathrm{CH}_{3} \mathrm{NH}_{3}+$ cations in the orthorhombic unit cell. Initial imparted energy is 164 meV (per unit cell). c Variation in the $\mathrm{C}-\mathrm{N}$ bond angle for different, indicated amounts of energy imparted into the unit cell. The purple curve is for a representative $\mathrm{C}-\mathrm{N}$ bond; orange and brown curves are data averaged over all of the four $\mathrm{CH}_{3} \mathrm{NH}_{3}+$ cations in the orthorhombic unit cell. Data in (b, c) are offset for clarity. $\mathbf{d}$ Transient $\Delta \mathrm{OD}$ spectra acquired at several different delay times; inset shows the overlapped transient spectra in arbitrary units by simple scaling. Fluence used was 0.45 $\mathrm{mJ} \mathrm{cm}{ }^{-2}$

following the selective excitation of N-H-as modes, phonon-phonon scattering leads to the sequential population of various lower-energy $\mathrm{CH}_{3} \mathrm{NH}_{3}{ }^{+}$vibrational modes, and ultimately the population of inorganic phonon modes. Note that thermal equilibration within the inorganic sublattice itself requires only 10 to $20 \mathrm{ps}$, as shown by ultrafast electron diffraction measurements ${ }^{4}$. Therefore, the nanosecond timescale is mainly attributed to the following two consecutive processes: (i) intramolecular vibrational relaxation (IVR) within the $\mathrm{CH}_{3} \mathrm{NH}_{3}{ }^{+}$ cations $^{34}$, and (ii) non-equilibrium thermal energy transfer from $\mathrm{CH}_{3} \mathrm{NH}_{3}{ }^{+}$to the inorganic framework ${ }^{31}$. The IVR process of the $\mathrm{CH}_{3} \mathrm{NH}_{3}{ }^{+}$cations involves a succession of energy downconversion through all the intermediate phonon modes starting from the selectively excited organic mode to the lowest-lying modes, which is expected to take longer than the lifetime of any specific organic vibrational mode. Indeed, several twodimensional infrared spectroscopic studies demonstrated a fewpicosecond timescale for the reorientational motions of the organic cations, as well as a significantly longer timescale (tens to hundreds of picoseconds) that was attributed to thermal response of the lattice ${ }^{13-15}$. Although the longer timescale has not been further pursed in those studies, we speculate that it is of similar origin as what we observed here. We note that the slow timescale can also be contributed by an ineffective (i.e., slow) heat energy transfer from the organic vibrational modes to the inorganic phonon modes, evident from the low thermal conductivities of organic-inorganic hybrid material systems ${ }^{35,36}$. We also note that the coupling between the high-energy vibrations and the electronic states during the first $10 \mathrm{ps}$ cannot be carefully addressed due to the excitation of optical phonon modes of the lead iodide framework (as discussed in the Time-resolved photoluminescence measurements section).

To gain more insights into the vibrational evolution, we performed ab initio molecular dynamics simulations on the orthorhombic structure with selective excitation of the N-H-as modes based on the phonon calculations, from which we extracted atomic trajectories up to 130 ps. As shown in Fig. 3b, selective excitation of the N-H-as modes results in the population of various stretching modes as represented by the variation of bond lengths of $\mathrm{N}-\mathrm{H}, \mathrm{C}-\mathrm{H}$, and $\mathrm{C}-\mathrm{N}$. The $\mathrm{C}-\mathrm{N}$ bond-length variation decays more slowly in comparison to the $\mathrm{C}-\mathrm{H}$ and $\mathrm{N}-\mathrm{H}$ bonds, as the latter two are associated with vibrational modes of the highest frequencies and depopulate most rapidly via IVR. Note that librational motions, captured by the orientational variation of the $\mathrm{C}-\mathrm{N}$ bond (Fig. 3c), can also be induced. The simulated timescale is furthermore not strongly dependent on the total imparted energy in the simulations (Fig. 3c), which is consistent with the experimental results (Fig. 2d).

The change of the $\Delta \mathrm{OD}$ amplitude can act as a proxy for how much thermal energy has been transferred to the inorganic framework (that in turn determines the bandgap). The transient response at long delay time (later than $1000 \mathrm{ps),} \mathrm{when} \mathrm{the} \mathrm{sample}$ has reached thermal equilibrium (Fig. 2b), results from the change of the bandgap with temperature. Transient spectra plotted for representative delay times (Fig. 3d) during the thermal relaxation closely overlap with a simple scaling of the amplitude. Such spectral similarity suggests that no net increase or decrease of optical absorption coefficient, other than that caused by lattice heating, occurs upon this perturbation when $\mathrm{CH}_{3} \mathrm{NH}_{3} \mathrm{PbI}_{3}$ is at thermal non-equilibrium (i.e., 0 to $1000 \mathrm{ps).} \mathrm{Because} \mathrm{optical}$ 
a

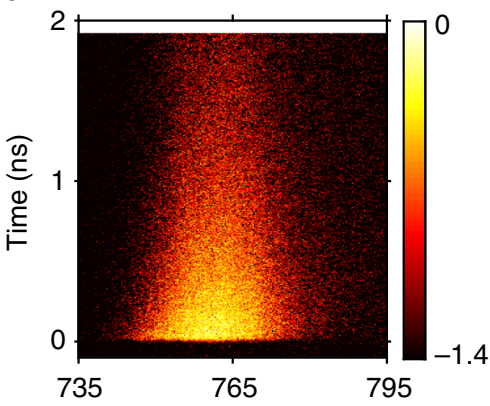

Wavelength $(\mathrm{nm})$

e

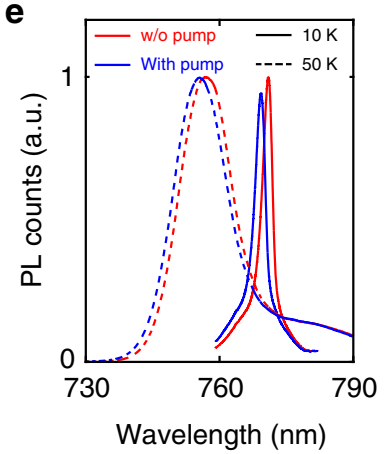

b

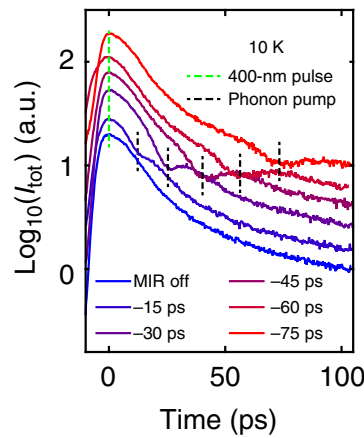

C

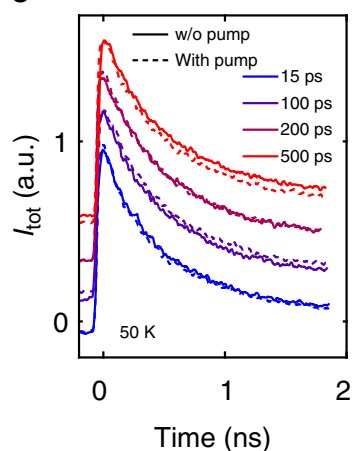

d

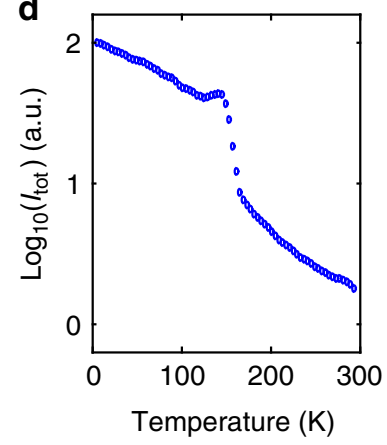

f

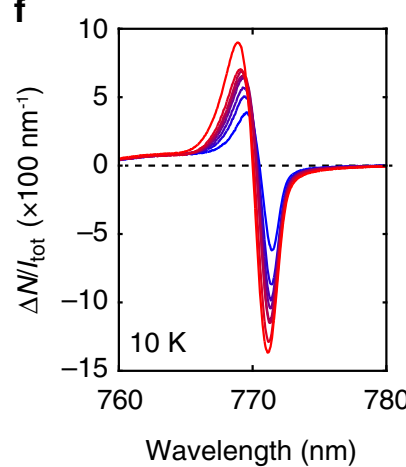

g

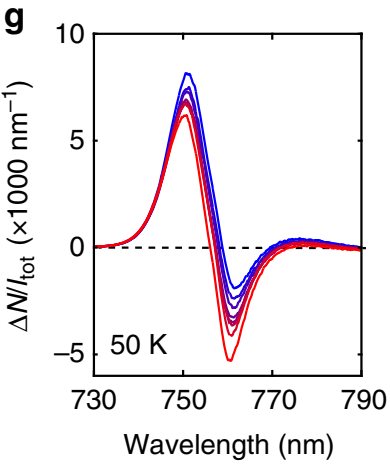

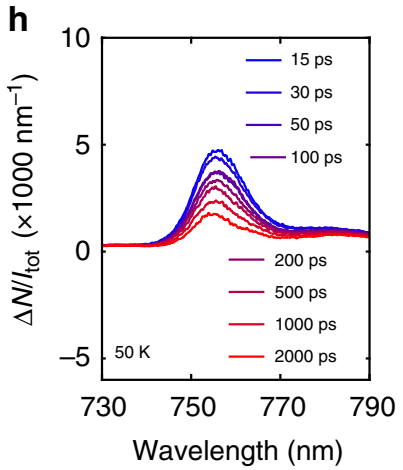

Fig. 4 Infrared pump photoluminescence (PL)-probe measurements of $\mathrm{CH}_{3} \mathrm{NH}_{3} \mathrm{Pbl}_{3}$. a Spectrally and temporally resolved $\mathrm{PL}$ counts measured with a streak camera (scalebar is in $\log _{10}$ scale) without using the infrared pump. b Decay kinetics of the PL intensity acquired under various negative $\Delta t$ values. $\mathbf{c}$ Decay kinetics of the PL intensity acquired under various positive $\Delta t$ values, with (dashed lines) and without (solid lines) using the infrared pump. $\mathbf{d}$ Static, temperature-dependent, wavelength-integrated PL intensity of the $\mathrm{CH}_{3} \mathrm{NH}_{3} \mathrm{Pbl}_{3}$ film. e Time-integrated PL spectra acquired at $10 \mathrm{~K}$ and at $50 \mathrm{~K}$, with $\Delta t=$ 2000 ps. Relative change of the time-integrated PL spectra with various positive delay times measured at $10 \mathrm{~K}$ in (f), and measured at $50 \mathrm{~K}$ in ( $\mathbf{g}$, $\mathbf{h}$ ). An on-resonance $\left(3120 \mathrm{~cm}^{-1}\right)$ infrared pump was used in $(\mathbf{e}, \mathbf{f}, \mathbf{g})$; an off-resonance $\left(2200 \mathrm{~cm}^{-1}\right)$ infrared pump was used in (h). Fluence of the infrared pump was fixed at $3.8 \mathrm{~mJ} \mathrm{~cm}^{-2}$ in these measurements. Legend in (h) also applies to $(\mathbf{f}, \mathbf{g})$

absorption can be viewed as an inverse process of bimolecular recombination $^{37}$, the data shown in Fig. $3 \mathrm{~d}$ implicitly suggest that the pump-induced $\mathrm{CH}_{3} \mathrm{NH}_{3}{ }^{+}$motions do not impact bimolecular recombination in $\mathrm{CH}_{3} \mathrm{NH}_{3} \mathrm{PbI}_{3}$.

IPEP measurements on $\mathrm{CH}_{3} \mathrm{NH}_{3} \mathrm{PbBr}_{3}$ and $\mathrm{CH}_{3} \mathrm{NH}_{3} \mathrm{PbCl}_{3}$ single crystals reveal similar transient optical response (Supplementary Fig. 5) compared to $\mathrm{CH}_{3} \mathrm{NH}_{3} \mathrm{PbI}_{3}$ due to their structural similarity. However, in contrast to $\mathrm{CH}_{3} \mathrm{NH}_{3} \mathrm{PbX}_{3}(\mathrm{X}=\mathrm{I}, \mathrm{Br}, \mathrm{Cl})$, which are three-dimensional (3D) perovskites, the impact of artificially induced organic cation motion is observed for the twodimensional perovskite, $\left(\mathrm{CH}_{3}\left(\mathrm{CH}_{2}\right)_{3} \mathrm{NH}_{3}\right)_{2} \mathrm{PbI}_{4}$. For this compound ${ }^{38}$, the $\mathrm{CH}_{3}\left(\mathrm{CH}_{2}\right)_{3} \mathrm{NH}_{3}{ }^{+}$cations situated between the perovskite layers strongly impact the electronic properties through both quantum and dielectric confinement effects. Following selective vibrational excitation near $3100 \mathrm{~cm}^{-1}$ (Supplementary Fig. 6), a much faster transient optical response, relative to the $3 \mathrm{D}$ counterparts, was observed for $\left(\mathrm{CH}_{3}\left(\mathrm{CH}_{2}\right)_{3} \mathrm{NH}_{3}\right)_{2} \mathrm{PbI}_{4}$ near the exciton transition energy (Supplementary Fig. 5), owing to the internal IVR processes of the $\mathrm{CH}_{3}\left(\mathrm{CH}_{2}\right)_{3} \mathrm{NH}_{3}{ }^{+}$cations that alter the strength of quantum confinement of the electron-hole pairs (i.e., excitons) residing on the $\mathrm{Pb}-\mathrm{I}$ framework.

Time-resolved photoluminescence measurements. We further explored if the infrared pump-induced $\mathrm{CH}_{3} \mathrm{NH}_{3}{ }^{+}$motions can directly impact photoluminescence (PL) of $\mathrm{CH}_{3} \mathrm{NH}_{3} \mathrm{PbI}_{3}$, using infrared pump PL-probe measurements based on the schematic shown in Supplementary Fig. 7. Here, the pump pulse was followed by a time-delayed (denoted as $\Delta t$ ), low-intensity, $400 \mathrm{~nm}$
PL-probe pulse that generates electron-hole pairs (positive $\Delta t$ is defined if the infrared pump hits the sample first). The emitted photons were either detected by a charge-coupled device (CCD) camera or spectrally and temporally resolved by a streak camera. Figure 4a presents a representative, spectrally resolved PL map measured at $50 \mathrm{~K}$ without using the infrared pump. Figure $4 \mathrm{~b}$ shows the spectrally integrated PL decay kinetics measured under various negative values of $\Delta t$, with the infrared pump turned on. The dip in the PL decay trace, which exhibits an instrumentresponse-time-limited timescale, is also observed by using an offresonant mid-infrared pump (Supplementary Fig. 8), and therefore attributed to the electric-field-driven dissociation of electron-hole pairs, or the optical Stark effect. We then focused on measurements under positive $\Delta t$ values. Figure $4 \mathrm{c}$ presents $\mathrm{PL}$ decays measured using different positive $\Delta t$ values that traverse the duration of sublattice thermal equilibration. We find that optically pumping the organic vibrational modes of $\mathrm{CH}_{3} \mathrm{NH}_{3} \mathrm{PbI}_{3}$ does not apparently alter the carrier recombination rate.

While streak camera measurements only detect PL over specific time windows, time-integrated PL measurements with higher signal-to-noise ratio can reveal whether the infrared pumpinduced $\mathrm{CH}_{3} \mathrm{NH}_{3}{ }^{+}$motions can enhance (by suppressing defect scattering of charge carriers) or suppress (by slowing down the recombination rate of carriers so that more trapping events can take place) the brightness of $\mathrm{CH}_{3} \mathrm{NH}_{3} \mathrm{PbI}_{3}$. Static, temperaturedependent PL intensity (Fig. 4d) suggests that a substantial portion of photo-generated carriers are lost through nonradiative decay pathways at $50 \mathrm{~K}$. The time-integrated PL spectra measured at $10 \mathrm{~K}$ and $50 \mathrm{~K}$ are shown in Fig. 4e; note that the dramatic change in PL linewidth upon temperature reduction has 
been observed previously ${ }^{39}$. For both temperatures, the infrared pump $(\Delta t=2000 \mathrm{ps})$ yields a blueshift of the PL spectrum, stemming from a bandgap increase due to lattice heating. We ran measurements at these two temperatures because the fast PL decay component due to bimolecular recombination ${ }^{40}$ is commensurate with the thermal equilibration time, and hence the impact of non-equilibrium $\mathrm{CH}_{3} \mathrm{NH}_{3}+$ motions, if there are any, can be more pronounced. Based on the PL spectral shift at $50 \mathrm{~K}$ (in conjunction with data in Fig. 1a and heat capacity ${ }^{41}$ ), we estimate that 0.06 photons are absorbed per orthorhombic unit cell by $\mathrm{CH}_{3} \mathrm{NH}_{3}{ }^{+}$, with a photon energy of $386 \mathrm{meV}$ (corresponding to $3120 \mathrm{~cm}^{-1}$ ). We denote the change of PL counts at each wavelength as $\Delta N=N_{\text {on }}-N_{\text {off }}$, where $N_{\text {on }}\left(N_{\text {off }}\right)$ designates PL counts obtained with (without) the infrared pump. The spectra of $\Delta N / I_{\text {tot }}$ measured at $10 \mathrm{~K}$ and $50 \mathrm{~K}$ using variable positive $\Delta t$ values are presented in Fig. $4 \mathrm{f}$, $\mathrm{g}$, respectively, where $I_{\text {tot }}$ is the spectrally integrated, total PL counts measured without the infrared pump. At $10 \mathrm{~K}$, infrared pumping yields a PL blueshift, the magnitude of which increases with $\Delta t$. This arises because a larger $\Delta t$ results in a more substantial sample heating (which causes a larger bandgap increase) before the photoluminescence event takes place. A similar PL blueshift is observed also at $50 \mathrm{~K}$, although an additional increase of the PL intensity, implied by an asymmetric line shape, is observed especially for small $\Delta t$ values. To explore the origin of such PL enhancement, we ran control experiments with an off-resonance infrared pump centered at $2200 \mathrm{~cm}^{-1}$. As shown in Fig. $4 \mathrm{~h}$, although the PL blueshift is no longer present due to the lack of lattice heating, a PL intensity enhancement is still recovered. The observed PL enhancement retained using both pumping wavelengths is likely attributable to polaron formation arising from below-bandgap excitation of various phonon modes of the inorganic sublattice (evident from Supplementary Fig. 9) ${ }^{6}$ that may contribute to the protection of charge carriers from defect scattering. This effect is not clearly observable at $10 \mathrm{~K}$ since the $\Delta N / I_{\text {tot }}$ intensity arising from bandgap blueshift is an order of magnitude larger than in the $50 \mathrm{~K}$ measurement. Importantly, comparison of Fig. $4 \mathrm{~g}, \mathrm{~h}$ reveals that the induced $\mathrm{CH}_{3} \mathrm{NH}_{3}{ }^{+}$motions alone lead to negligible variation of the integrated PL intensity.

\section{Discussion}

Our transient absorption and PL measurements both suggest that infrared pump-induced $\mathrm{CH}_{3} \mathrm{NH}_{3}{ }^{+}$motions do not lead to noticeable changes of the near-bandgap absorption and $\mathrm{PL}$ properties, beyond effects due to lattice heating. Such an observation implies that photo-generated carriers residing on the inorganic sublattice are essentially decoupled from the dynamically disordered organic cations. The transient spectroscopic technique reported here, complementary to fully electronic pump-probe, or fully infrared (vibrational) pump-probe measurements ${ }^{14,42,43}$, can be generalized for studying organic semiconductors, organic-inorganic interfaces, and other organic-inorganic hybrid materials such as superatomic solids ${ }^{35}$ and two-dimensional hybrid perovskites ${ }^{44}$.

\footnotetext{
Methods

Chemicals and the synthesis of $\mathbf{C H}_{\mathbf{3}} \mathbf{N H}_{\mathbf{3}} \mathbf{l}$. Methylamine solution $\left(\mathrm{CH}_{3} \mathrm{NH}_{2}\right.$, 40 wt.\% in $\mathrm{H}_{2} \mathrm{O}$ ), $\mathrm{N}$-methyl-2-pyrrolidinone (NMP), and $\gamma$-Butyrolactone (GBL, $\geq 99 \%$ ) were purchased from Aldrich. Hydriodic acid ( $\mathrm{HI}, 57 \mathrm{wt} . \%$ in $\mathrm{H}_{2} \mathrm{O}$ ) and lead(II) iodide $\left(\mathrm{PbI}_{2}, 99.9985 \%\right.$ metal basis) were purchased from Alfa Aesar. Ethyl ether (anhydrous) and acetone were obtained from Fisher Chemical. All chemicals were used as received without further purification. Equimolar of HI was dropwise added to $\mathrm{CH}_{3} \mathrm{NH}_{2}$ with stirring in a $50 \mathrm{~mL}$ round bottom flask immersed in ice bath, followed by rotary evaporation at $60^{\circ} \mathrm{C}$ to dry off the solvent. Next, the white solid was obtained and washed with excessive ethyl ether on a filter paper, accompanied with vacuum filtration. The washed $\mathrm{CH}_{3} \mathrm{NH}_{3} \mathrm{I}$ powder was then dried in a vacuum oven at $60^{\circ} \mathrm{C}$ overnight to yield the final product.
}

$\mathbf{C H}_{\mathbf{3}} \mathbf{N H}_{\mathbf{3}} \mathbf{P b l}_{\mathbf{3}}$ thin film fabrication. The preparation of $\mathrm{CH}_{3} \mathrm{NH}_{3} \mathrm{PbI}_{3}$ thin film follows method reported previously with slight modification ${ }^{45}$. The 1.2:1 molar ratio of $\mathrm{CH}_{3} \mathrm{NH}_{3} \mathrm{I} / \mathrm{PbI}_{2}$ was dissolved in 1:1 volume ratio of NMP/GBL to make a $40 \mathrm{wt} . \%$ precursor solution. Then, the precursor solution was spun-coated on an acetone-cleaned sapphire substrate with a speed of $3000 \mathrm{rpm}$ for $30 \mathrm{~s}$. Next, the asformed wet film was quickly transferred into an ethyl ether bath $(50 \mathrm{~mL})$ for $90 \mathrm{~s}$ Finally, the developed film was dried in air, covered by a Petri dish, and followed by annealing at $150^{\circ} \mathrm{C}$ for $15 \mathrm{~min}$ on a hot plate in a humidity-controlled environment.

Static optical characterization. Static infrared absorbance spectra were captured with Fourier transform infrared (FTIR; Thermo Nicolet 6700). Static visible absorbance spectra were obtained with a customized setup. In all the optical measurements (both static and transient), the samples were mounted in a $4 \mathrm{~K}$ closed-cycle cold-finger cryostat under with a base pressure below $1 \times 10^{-7}$ Torr.

Transient absorption measurements. Transient absorption (i.e., IPEP) measurements were performed using a $35 \mathrm{fs}$ amplified titanium/sapphire laser operating at $800 \mathrm{~nm}$ at a repetition rate of $2 \mathrm{kHz}$. Broadband visible probe pulses were generated by focusing a portion of the amplifier output onto an $\mathrm{Al}_{2} \mathrm{O}_{3}$ window. Infrared pump pulses were generated by difference frequency mixing of signal and idler beams using an optical parametric amplifier and were reduced in repetition rate to $1 \mathrm{kHz}$. The probe pulses were mechanically time delayed using a translation stage and retroreflector. Full transient spectral maps are shown in Supplementary Figs. 10 and Fig. 11.

Infrared pump PL-probe measurements. Generation of the infrared pump pulse was identical to that employed in the transient absorption measurements. The 400 $\mathrm{nm}$ PL excitation pulses were produced by frequency-doubling of the $800 \mathrm{~nm}$ amplifier output with a BBO crystal and underwent similar beam path as the visible probe pulses in the IPEP measurements. Both the infrared pump pulses and the $400 \mathrm{~nm}$ PL excitation pulses were maintained at $2 \mathrm{kHz}$. Temporally and spectrally resolved PL data were collected with a streak camera. Time-integrated PL data were captured with a CCD camera. The spot size of the 400-nm PL excitation pulses was adjusted to be smaller than that of the infrared pump pulses. The fluence of $400 \mathrm{~nm}$ PL excitation pulse was kept below $1 \mu \mathrm{J} \mathrm{cm}^{-2}$.

\section{Calculations of the phonon spectrum and phonon-phonon scattering phase} space. Vienna Ab initio Simulation Package (VASP) was used to perform structure relaxation and self-consistent density functional theory (DFT) calculations ${ }^{46-49}$. The projector augmented wave (PAW) method ${ }^{50}$ was used in conjunction with the generalized gradient approximation $(\mathrm{GGA})^{51}$ for the exchange-correlation $(\mathrm{xc})$ functional ${ }^{52}$. We used the van der Waals density functional (vdW-DF) method to treat the dispersion forces from non-local electron-electron interactions, particularly, the optB86b-vdW functional ${ }^{53}$. DFT calculations of primitive cell of $\mathrm{CH}_{3} \mathrm{NH}_{3} \mathrm{PbI}_{3}$ in the orthorhombic phase with space group of Pnma (No. 62) were performed using a $4 \times 4 \times 4 \Gamma$-centered $\mathbf{k}$-point mesh and a kinetic energy cutoff of $550 \mathrm{eV}$. The force and energy convergence thresholds were set to be $5 \times 10^{-3} \mathrm{eV}^{-1}$ and $10^{-8} \mathrm{eV}$, respectively. Phonon calculations were performed using Phonopy package ${ }^{54}$, wherein $1 \times 1 \times 1$ supercell structures were constructed to extract harmonic interatomic force constants which were used to construct dynamical matrix and obtain phonon dispersion relation along high-symmetry paths in the Brillouin zone. Phonon scattering phase space accounting for three-phonon interactions was calculated using ShengBTE package ${ }^{55}$.

Ab initio molecular dynamics (AIMD) simulations. The starting structures for AIMD were obtained by exciting all the $\mathrm{CH}_{3} \mathrm{NH}_{3}+$ cations by a certain fraction of the phonon eigenvectors for the highest energy mode (the N-H-as mode). To study the effect of fluence on the perovskite dynamics, the atoms in the $\mathrm{CH}_{3} \mathrm{NH}_{3}{ }^{+}$ cations were displaced from their equilibrium positions by a fraction of 0.10 and 0.07 , which correspond to an energy imparted of $164 \mathrm{meV}$ and $72 \mathrm{meV}$, respectively. AIMD simulations were performed as implemented in the VASP package, using PAW pseudopotentials and the GGA functional with vdW-DF correction. Because of computational expense, spin-orbit coupling was not considered. An energy cutoff of $500 \mathrm{eV}$ was used for the plane wave basis. The atoms were relaxed until forces on the atoms were less than $0.0001 \mathrm{eV}^{-1}$, and gamma point sampling was used for the Brillouin zone. A time step of $0.5 \mathrm{fs}$ was applied for the AIMD calculations. Standard molecular dynamics was performed using a microcanonical ensemble by applying the IBRION $=0$ and the SMASS $=-3$ tags. The AIMD trajectories and structure snapshots were visualized with VESTA and VMD softwares ${ }^{56,57}$. The structure of the $\mathrm{CH}_{3} \mathrm{NH}_{3} \mathrm{PbI}_{3}$ used in the calculation was obtained from ref. 58 .

\section{Data availability}

All relevant data used in the article are available from the authors. 
Received: 11 September 2018 Accepted: 28 December 2018

Published online: 29 January 2019

\section{References}

1. Lee, M. M., Teuscher, J., Miyasaka, T., Murakami, T. N. \& Snaith, H. Efficient hybrid solar cells based on meso-superstructured organometal halide perovskites. Science 338, 643-647 (2012).

2. Jia, Y., Kerner, R. A., Grede, A. J., Rand, B. P. \& Giebink, N. C. Continuouswave lasing in an organic-inorganic lead halide perovskite semiconductor. Nat. Photonics 11, 784-788 (2017).

3. Xing, G. et al. Long-range balanced electron- and hole-transport lengths in organic-inorganic $\mathrm{CH}_{3} \mathrm{NH}_{3} \mathrm{PbI}_{3}$. Science 342, 344-347 (2013).

4. $\mathrm{Wu}, \mathrm{X}$. et al. Light-induced picosecond rotational disordering of the inorganic sublattice in hybrid perovskites. Sci. Adv. 3, e1602388 (2017).

5. Yaffe, O. et al. Local polar fluctuations in lead halide perovskite crystals. Phys. Rev. Lett. 118, 136001 (2017).

6. Miyata, K. et al. Large polarons in lead halide perovskites. Sci. Adv. 3, e1701217 (2017).

7. Leguy, A. M. A. et al. The dynamics of methylammonium ions in hybrid organic-inorganic perovskite solar cells. Nat. Commun. 6, 7124 (2015).

8. Poglitsch, A. \& Weber, D. Dynamic disorder in methylammoniumtrihalogenoplumbates (II) observed by millimeter-wave spectroscopy. J. Chem. Phys. 87, 6373-6378 (1987).

9. Wang, L., McCleese, C., Kovalsky, A., Zhao, Y. \& Burda, C. Femtosecond time-resolved transient absorption spectroscopy of $\mathrm{CH}_{3} \mathrm{NH}_{3} \mathrm{PbI}_{3}$ perovskite films: evidence for passivation effect of $\mathrm{PbI}_{2}$. J. Am. Chem. Soc. 136, 12205-12208 (2014).

10. Odenthal, P. et al. Spin-polarized exciton quantum beating in hybrid organic-inorganic perovskites. Nat. Phys. 13, 894-899 (2017).

11. Ye, H.-Y. et al. Metal-free three-dimensional perovskite ferroelectrics. Science 361, 151-155 (2018).

12. Frost, J. M. et al. Atomistic origins of high-performance in hybrid halide perovskite solar cells. Nano Lett. 14, 2584-2590 (2014).

13. Taylor, V. C. A. et al. Investigating the role of the organic cation in formamidinium lead iodide perovskite using ultrafast spectroscopy. J. Phys. Chem. Lett. 9, 895-901 (2018).

14. Bakulin, A. A. et al. Real-time observation of organic cation reorientation in methylammonium lead iodide perovskites. J. Phys. Chem. Lett. 6, 3663-3669 (2015).

15. Selig, O. et al. Organic cation rotation and immobilization in pure and mixed methylammonium lead-halide perovskites. J. Am. Chem. Soc. 139, 4068-4074 (2017).

16. Motta, C. et al. Revealing the role of organic cations in hybrid halide perovskite $\mathrm{CH}_{3} \mathrm{NH}_{3} \mathrm{PbI}_{3}$. Nat. Commun. 6, 7026 (2015).

17. Zheng, F., Tan, L. Z., Liu, S. \& Rappe, A. M. Rashba spin-orbit coupling enhanced carrier lifetime in $\mathrm{CH}_{3} \mathrm{NH}_{3} \mathrm{PbI}_{3}$. Nano Lett. 15, 7794-7800 (2015).

18. Frohna, K. et al. Inversion symmetry and bulk Rashba effect in methylammonium lead iodide perovskite single crystals. Nat. Commun. 9, 1829 (2018).

19. Yang, W. S. et al. High-performance photovoltaic perovskite layers fabricated through intramolecular exchange. Science 348, 1234-1237 (2015).

20. Zhu, H. et al. Organic cations might not be essential to the remarkable properties of band edge carriers in lead halide perovskites. Adv. Mater. 29, 1603072 (2017).

21. Kulbak, M., Cahen, D. \& Hodes, G. How important is the organic part of lead halide perovskite photovoltaic cells? Efficient $\mathrm{CsPbBr}_{3}$ cells. J. Phys. Chem. Lett. 6, 2452-2456 (2015).

22. Zhu, H. et al. Screening in crystalline liquids protects energetic carriers in hybrid perovskites. Science 353, 1409-1413 (2016).

23. Wu, X. et al. Trap states in lead iodide perovskites. J. Am. Chem. Soc. 137, 2089-2096 (2015).

24. Stoumpos, C. C. \& Kanatzidis, M. G. The renaissance of halide perovskites and their evolution as emerging semiconductors. Acc. Chem. Res. 48, 2791-2802 (2015).

25. Hutter, E. M. et al. Vapour-deposited cesium lead iodide perovskites: microsecond charge carrier lifetimes and enhanced photovoltaic performance. ACS Energy Lett. 2, 1901-1908 (2017).

26. Wang, P. et al. Solvent-controlled growth of inorganic perovskite films in dry environment for efficient and stable solar cells. Nat. Commun. 9, 2225 (2018).

27. Sanehira, E. M. et al. Enhanced mobility $\mathrm{CsPbI}_{3}$ quantum dot arrays for record-efficiency, high-voltage photovoltaic cells. Sci. Adv. 3, eaao4204 (2017).

28. Egger, D. A. et al. What remains unexplained about the properties of halide perovskites? Adv. Mater. 30, 1800691 (2018).
29. Fu, Y. et al. Selective stabilization and photophysical properties of metastable perovskite polymorphs of $\mathrm{CsPbI}_{3}$ in thin films. Chem. Mater. 29, 8385-8394 (2017).

30. Grechko, M., Bretschneider, S. A., Vietze, L., Kim, H. \& Bonn, M. Vibrational coupling between organic and inorganic sublattices of hybrid perovskites. Angew. Chem. Int. Ed. 57, 13657-13661 (2018).

31. Guo, P. et al. Slow thermal equilibration in methylammonium lead iodide revealed by transient mid-infrared spectroscopy. Nat. Commun. 9, 2792 (2018).

32. Whitfield, P. S. et al. Structures, phase transitions and tricritical behavior of the hybrid perovskite methyl ammonium lead iodide. Sci. Rep. 6, 35685 (2016).

33. Yang, Y. et al. Large polarization-dependent exciton optical Stark effect in lead iodide perovskites. Nat. Commun. 7, 12613 (2016).

34. Elles, C. G., Cox, M. J. \& Crim, F. F. Vibrational relaxation of $\mathrm{CH}_{3} \mathrm{I}$ in the gas phase and in solution. J. Chem. Phys. 120, 6973-6979 (2004).

35. Ong, W.-L. et al. Orientational order controls crystalline and amorphous thermal transport in superatomic crystals. Nat. Mater. 16, 83-88 (2016).

36. Ong, W.-L., Rupich, S. M., Talapin, D. V., McGaughey, A. J. H. \& Malen, J. A. Surface chemistry mediates thermal transport in three-dimensional nanocrystal arrays. Nat. Mater. 12, 410-415 (2013).

37. Davies, C. L. et al. Bimolecular recombination in methylammonium lead triiodide perovskite is an inverse absorption process. Nat. Commun. 9, 293 (2018).

38. Stoumpos, C. C. et al. Ruddlesden-Popper hybrid lead iodide perovskite 2D homologous semiconductors. Chem. Mater. 28, 2852-2867 (2016).

39. Wright, A. D. et al. Electron-phonon coupling in hybrid lead halide perovskites. Nat. Commun. 7, 11755 (2016).

40. Milot, R. L., Eperon, G. E., Snaith, H. J., Johnston, M. B. \& Herz, L. M. Temperature-dependent charge-carrier dynamics in $\mathrm{CH}_{3} \mathrm{NH}_{3} \mathrm{PbI}_{3}$ perovskite thin films. Adv. Funct. Mater. 25, 6218-6227 (2015).

41. Onoda-Yamamuro, N., Matsuo, T. \& Suga, H. Calorimetric and IR spectroscopic studies of phase transitions in methylammonium trihalogenoplumbates (II)†. J. Phys. Chem. Solids 51, 1383-1395 (1990).

42. Guo, Z. et al. Long-range hot-carrier transport in hybrid perovskites visualized by ultrafast microscopy. Science 356, 59-62 (2017).

43. Guo, P. et al. Cross-plane coherent acoustic phonons in two-dimensional organic-inorganic hybrid perovskites. Nat. Commun. 9, 2019 (2018).

44. Smith, M. D. \& Karunadasa, H. I. White-light emission from layered halide perovskites. ACC Chem. Res. 51, 619-627 (2018).

45. Yang, M. et al. Square-centimeter solution-processed planar $\mathrm{CH}_{3} \mathrm{NH}_{3} \mathrm{PbI}_{3}$ perovskite solar cells with efficiency exceeding $15 \%$. Adv. Mater. 27, 6363-6370 (2015)

46. Kresse, G. \& Hafner, J. Ab initio molecular dynamics for liquid metals. Phys. Rev. B 47, 558-561 (1993).

47. Kresse, G. \& Hafner, J. Ab initio molecular-dynamics simulation of the liquidmetal--amorphous-semiconductor transition in germanium. Phys. Rev. B 49, 14251-14269 (1994).

48. Kresse, G. \& Furthmüller, J. Efficiency of ab-initio total energy calculations for metals and semiconductors using a plane-wave basis set. Comput. Mater. Sci. 6, 15-50 (1996).

49. Kresse, G. \& Furthmüller, J. Efficient iterative schemes for ab initio totalenergy calculations using a plane-wave basis set. Phys. Rev. B 54, 11169-11186 (1996).

50. Blöchl, P. E. Projector augmented-wave method. Phys. Rev. B 50, 17953-17979 (1994).

51. Perdew, J. P., Burke, K. \& Wang, Y. Generalized gradient approximation for the exchange-correlation hole of a many-electron system. Phys. Rev. B 54, 16533-16539 (1996).

52. Hohenberg, P. \& Kohn, W. Inhomogeneous electron gas. Phys. Rev. 136, B864-B871 (1964).

53. Klimeš, J., Bowler, D. R. \& Michaelides, A. Van der Waals density functionals applied to solids. Phys. Rev. B 83, 195131 (2011).

54. Togo, A. \& Tanaka, I. First principles phonon calculations in materials science. Scr. Mater. 108, 1-5 (2015).

55. Li, W., Carrete, J., A. Katcho, N. \& Mingo, N. ShengBTE: a solver of the Boltzmann transport equation for phonons. Comput. Phys. Commun. 185, $1747-1758$ (2014).

56. Momma, K. \& Izumi, F. VESTA 3 for three-dimensional visualization of crystal, volumetric and morphology data. J. Appl. Crystallogr. 44, 1272-1276 (2011).

57. Humphrey, W., Dalke, A. \& Schulten, K. VMD: Visual molecular dynamics. J. Mol. Graph. 14, 33-38 (1996).

58. Brivio, F. et al. Lattice dynamics and vibrational spectra of the orthorhombic, tetragonal, and cubic phases of methylammonium lead iodide. Phys. Rev. B 92, 144308 (2015). 


\section{Acknowledgements}

The work was performed, in part, at the Center for Nanoscale Materials, a U.S. Department of Energy Office of Science User Facility, and supported by the U.S. Department of Energy, Office of Science, under Contract No. DE-AC02-06CH11357. This material is based upon work supported by Laboratory Directed Research and Development (LDRD) funding from Argonne National Laboratory, provided by the Director, Office of Science, of the U.S. Department of Energy under contract DE-AC0206CH11357. Work at Northwestern University was supported by grant SC0012541 from the US Department of Energy, Office of Science (sample preparation). T.X. acknowledges the financial support from National Science Foundation (DMR-1806152). We thank Dr. A. B. F. Martinson for comments on the paper.

\section{Author contributions}

P.G. conceived the infrared pump electronic-probe measurements under the supervision of R.D.S. J.G., C.C.S., D.H.C. and B.T.D. fabricated the samples under the supervision of T.X., M.K.G. and R.D.S. A.M.-K. and Y.X. performed first-principles calculations under the supervision of M.K.Y.C. G.P.W. and J.B.K. contributed to the experiments and analysis. P.G. wrote the manuscript with input from all authors. R.D.S. supervised the project.

\section{Additional information}

Supplementary Information accompanies this paper at https://doi.org/10.1038/s41467019-08363-2.

Competing interests: The authors declare no competing interests.
Reprints and permission information is available online at http://npg.nature.com/ reprintsandpermissions/

Journal peer review information: Nature Communications thanks Daniel Vanmaekelbergh and the other anonymous reviewer(s) for their contribution to the peer review of this work.

Publisher's note: Springer Nature remains neutral with regard to jurisdictional claims in published maps and institutional affiliations.

(c) (i) Open Access This article is licensed under a Creative Commons Attribution 4.0 International License, which permits use, sharing, adaptation, distribution and reproduction in any medium or format, as long as you give appropriate credit to the original author(s) and the source, provide a link to the Creative Commons license, and indicate if changes were made. The images or other third party material in this article are included in the article's Creative Commons license, unless indicated otherwise in a credit line to the material. If material is not included in the article's Creative Commons license and your intended use is not permitted by statutory regulation or exceeds the permitted use, you will need to obtain permission directly from the copyright holder. To view a copy of this license, visit http://creativecommons.org/ licenses/by/4.0/.

(C) This is a U.S. Government work and not under copyright protection in the US; foreign copyright protection may apply 2019 